\title{
Desgarros del epitelio pigmentario de la retina: factores de riesgo, mecanismo y control terapéutico
}

\author{
Christoph R. Clemens Nicole Eter \\ Departamento de Oftalmología, Universidad de Münster Medical Centre, Münster, Alemania
}

\section{Palabras clave}

Degeneración macular relacionada con la edad .

Epitelio pigmentario de la retina - Desprendimiento del epitelio pigmentario de la retina - Desgarro del epitelio pigmentario de la retina $\cdot$ Inyección intravítrea $\cdot$ Tomografía de coherencia óptica de dominio espectral

\section{Resumen}

Los desgarros del epitelio pigmentario de la retina (EPR) se asocian en la mayoría de los casos con los desprendimientos vascularizados del EPR debido a una degeneración macular asociada a la edad (DMAE), y normalmente implican una pérdida adversa de la agudeza visual. Estudios recientes indican que ha habido un aumento en la incidencia de desgarros del EPR desde la introducción de fármacos anti-factor de crecimiento del endotelio vascular (anti-VEGF) así como una asociación temporal entre el desgarro y la inyección intravítrea. Dado que el número de pacientes con DMAE y el número de inyecciones anti-VEGF va en aumento, tanto la dificultad de prevenir desgarros del EPR como el tratamiento tras la formación de los desgarros han adquirido una mayor relevancia. De forma paralela, la evolución de la imagenología de la retina ha contribuido de manera significativa a comprender mejor el desarrollo de los desgarros del EPR en los últimos años. Esta revisión resume los conocimientos que se poseen actualmente sobre el desarrollo, los factores pronósticos y las estrategias terapéuticas de los desgarros del EPR antes y después de que estos se formen.

๑) 2017 S. Karger AG, Basel

\section{Introducción}

La introducción de los fármacos anti-factor de crecimiento del endotelio vascular (anti-VEGF, por sus siglas en inglés) para el tratamiento de pacientes que presentan degeneración macular exudativa asociada a la edad sin duda ha resultado un gran avance en el ámbito de la oftalmología y, hasta ahora, ha evitado que se produzca pérdida de visión grave en un gran número de casos. Los desgarros del epitelio pigmentario de la retina (EPR) constituyen una complicación poco frecuente en el tratamiento de pacientes con degeneración macular exudativa relacionada con la edad y, a menudo, conlleva una pérdida grave de la agudeza visual [1-3].

Se sabe que los desgarros del EPR suceden como resultado natural en el transcurso de un desprendimiento

The Spanish version should be cited as DOI: 10.1159/000477514. The original version is in English and should be cited as DOI: $10.1159 / 000439445$.

\section{KARGER}

(C) 2017 S. Karger AG, Basel

E-Mail karger@karger.com

www.karger.com/oph
Christoph R. Clemens, MD

Departamento de Oftalmología, Universidad de Münster Medical Centre Domagkstrasse 15

DE-48149 Münster (Alemania)

E-Mail Christoph.Clemens@ukmuenster.de 
del epitelio pigmentario (DEP) de la retina debido a neovascularización coroidea subyacente (NVC), a proliferación angiomatosa de la retina o a vasculopatía coroidea polipoidal. Desde el comienzo de los tratamientos con anti-VEGF intravítreos en pacientes con DEP debido a degeneración macular exudativa relacionada con la edad, se ha venido informando de un mayor número de desgarros del EPR como complicación tras la inyección.

Dado que ha aumentado con total claridad el número de inyecciones, los médicos se enfrentan con más frecuencia a la situación de prevenir el desgarro del EPR o tener que tratarlo después de que se haya formado. La evolución de la imagenología de la retina ha contribuido significativamente a comprender mejor el desarrollo de los desgarros del EPR y ha demostrado ser un instrumento valioso a la hora de adaptar los regímenes terapéuticos en pacientes con DEP.

Esta revisión destaca los conocimientos de los que se disponen actualmente sobre el desarrollo, los factores pronósticos que indican un desgarro inminente del EPR, y las estrategias terapéuticas de los desgarros del EPR antes y después de que estos se formen.

\section{Epidemiología}

En 1981, Hoskin et al. documentaron por primera vez los desgarros del EPR [4] como complicación en pacientes con DEP debido a una degeneración macular asociada a la edad (DMAE). Los desgarros del EPR pueden ser parte del transcurso natural del DEP debido a una NVC inadvertida, a una proliferación angiomatosa de la retina, a una vasculopatía coroidea polipoidal, o pueden suceder en relación con varios tratamientos para la degeneración neovascular macular relacionada con la edad, tales como la terapia fotodinámica, la fotocoagulación con láser o la termoterapia transpupilar [5-7].

La incidencia se reporta entre el 5 y el $27 \%$ entre las personas de edad avanzada [1-21]. Casswell et al. [1] presentaron una tasa de desgarros espontáneos de los DEP vasculares del $10 \%$. En la rutina clínica actual, parece que la mayoría de los desgarros del EPR están estrechamente relacionados con tratamientos anti-VEGF y se ha informado de ello en el caso de pegaptanib, bevacizumab, ranibizumab y aflibercept [8-11]. La primera descripción de un desgarro del EPR posterior a la inyección intravítrea de bevacizumab para el tratamiento de NVC inadvertido debido a una DMAE fue realizada por Meyer et al. [12] en 2006.

Diferentes incidencias de desgarros del EPR se reportan con los distintos agentes de tratamiento y con los di-

Desgarros del EPR ferentes regímenes de tratamiento [13-22]. Un estudio retrospectivo de 1.280 ojos tratados con bevacizumab incluyó 125 ojos con DEP vascularizado (DEPv) mostrando una tasa de desgarros del EPR de 16,8 \% [13]. En una serie de casos de 6 pacientes con DEPv, Chang et al. [14] presentaron una incidencia de desgarro del $27 \%$ en tratamiento con pegaptanib. Clemens et al. [15] presentaron una incidencia de desgarro del $25 \%$ en un estudio prospectivo de un régimen mensual de ranibizumab en 40 pacientes con DEPv. Recientemente, Sarraf et al. [16] presentaron resultados de un estudio prospectivo aleatorizado sobre el tratamiento de ranibizumab de 0,5 ó $2,0 \mathrm{mg}$ sobre la base de un régimen mensual o cuando fuera necesario en ojos con DEPv que mostró una incidencia del $14 \%$ (5/37 pacientes). Se destaca como dato interesante que 4 de los 5 desgarros del EPR presentados sucedieron en el grupo de la dosis más alta. Al margen de unos pocos casos publicados, todavía no se disponen de datos prospectivos sobre la tasa de desgarros del EPR en pacientes con DEPv en tratamiento con aflibercept [11]. Existe un solo caso publicado sobre desarrollo de desgarro del EPR en ojo contralateral no tratado después de recibir antiVEGF para el tratamiento de la DMAE neovascular. La relación causante sigue siendo especulativa [23].

Las incidencias presentadas en publicaciones sobre desgarros del EPR deben interpretarse con precaución, pues los colectivos de pacientes subyacentes, los regímenes de tratamiento, así como la nomenclatura de la morfología de la lesión tienden a ser muy heterogéneos. No obstante, las incidencias superiores de desgarros del EPR en ojos en tratamiento anti-VEGF en comparación con los estudios de evolución natural en ojos con DEP indican que hay un efecto potenciador de los tratamientos anti-VEGF en el desarrollo del desgarro del EPR. Se presupone que en un futuro cercano no se llevarán a cabo estudios prospectivos comparativos debido a cuestiones éticas.

\section{Imagenología}

\section{Retinografía}

Con una oftalmoscopia se puede sospechar un desgarro del EPR sobre la base de la presencia de sangrado subretiniano y subEPR, una línea hiperpigmentada bien definida en el lugar del EPR enrollado, y un área despigmentada correspondiente a la coroides expuesta [4]. Las hemorragias retinianas o subretinianas se distinguen claramente de las áreas del desgarro del EPR en la retinografía. Es posible que las hemorragias interfieran a la hora de

Ophthalmologica 2017;238(suppl 1):28-38 DOI: $10.1159 / 000477514$ 

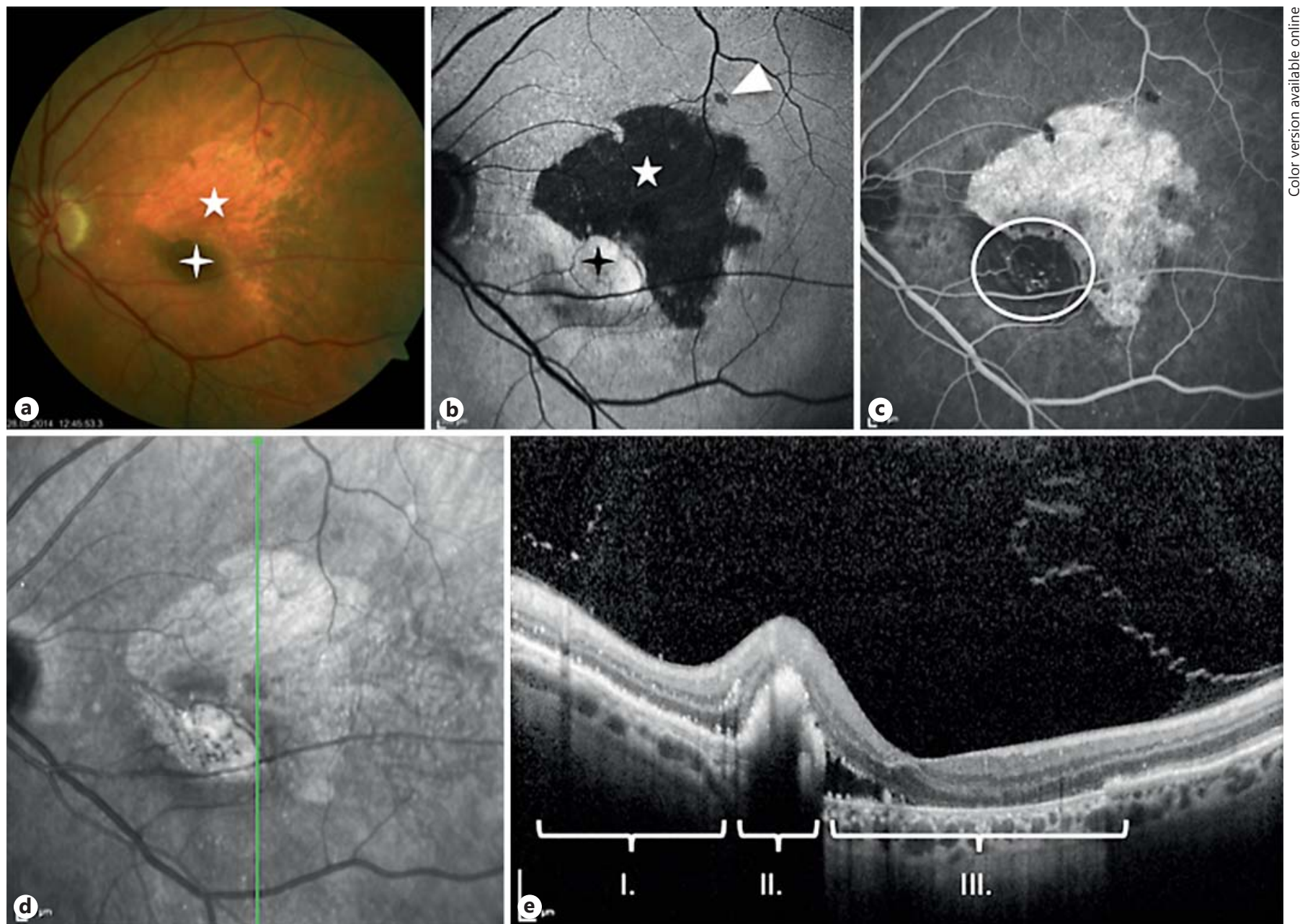

c.

Fig. 1. Imágenes multimodales de un paciente ejemplar con DEPv debido a una DMAE tras desarrollo del desgarro del EPR. a La retinografía muestra un área despigmentada correspondiente a la coroides expuesta (estrella) y un área hiperpigmentada correspondiente al EPR enrollado (diamante). Téngase en cuenta la pequeña hemorragia retiniana situada en la parte superior del desgarro del EPR, que no se distingue claramente del área hipoautofluorescente del desgarro en la modalidad de AFF como se muestra en b (punta de la flecha). b La imagen de AFF de la oftalmoscopia confocal láser de barrido muestra una gran y claramente perfilada señal hipoautofluorescente en el área del desgarro del EPR (estrella), así como un área hiperautofluorescente correspondiente al EPR

detectar el área del desgarro del EPR en la autofluorescencia de fondo (AFF) (fig. 1a, b), lo que hace que la retinografía sea indispensable en estos casos.

Sarraf et al. [24] introdujeron un sistema de calificación de desgarros del EPR con retinografía. Los desgarros de grado 1 se definieron como $<200 \mu \mathrm{m}$. Los desgarros de grado 2 estaban entre $200 \mu \mathrm{m}$ y 1 diámetro del disco. Los desgarros de grado 3 se consideraron con $>1$ diámetro del enrollado (diamante). c La angiografía con fluoresceína de fase tardía revela una NVC en la parte inferior de la lesión (círculo) y una señal hiperfluorescente en el área del desgarro. De forma notable, el desgarro del EPR ha sucedido en el lado opuesto a donde se localiza la NVC. d, e La imagen del infrarrojo cercano con oftalmoscopía confocal láser de barrido (d) combinada con la imagen de OCT SD (e) muestra una arquitectura normal de las bandas externas retinianas en la primera parte. En la segunda parte, el borde libre del desgarro del EPR se retrae hacia la NVC. Téngase en cuenta la banda del EPR ausente en el área del desgarro que representa la tercera parte.

disco. Los desgarros de grado 4 se definieron como desgarros de grado 3 que afectaban el centro de la fóvea.

\section{Autofluorescencia de fondo}

En las imágenes de la autofluorescencia de fondo (AFF), los desgarros del EPR muestran una señal autoflorescente notablemente reducida porque las células del EPR con lipofuscina autofluorescente se pierden [25]. 
Los desgarros pequeños del EPR son más notables en la AFF y resultan más fáciles de detectar que con la retinografía. El gran contraste de estas áreas hipoautofluorescentes, en comparación con la retina intacta, permite determinar de forma sencilla y precisa los límites de la lesión (fig. 1b). La configuración de los desgarros del EPR se clasifica como unilobular si las áreas del EPR están formadas de manera homogénea y como multilobular si los defectos en el EPR consisten en dos o más lóbulos separados por un fragmento de filamento del EPR intacto [26]. Con el tiempo, los bordes de hipoautofluorescencia se vuelven difusos y menos delimitados en la AFF. Sin embargo, los primeros cambios morfológicos que se observan en los bordes de los desgarros del EPR mediante $\mathrm{AFF}$, descritos como puntos hiperreflectivos de varios diámetros, no se ven hasta 3 meses después del evento agudo de la formación del desgarro del EPR. Los procesos de remodelación en la zona limítrofe no parecen ser detectables por imágenes de AFF durante los primeros meses [27]. En comparación con la atrofia geográfica en la DMAE, las áreas hipofluorescentes en la AFF debido a la formación de desgarro del EPR no muestran patrones de incremento anormal de la AFF en la zona de la unión de la atrofia [28].

\section{Angiografía fluoresceínica}

La angiografía fluoresceínica (AGF) muestra bloqueo de la fluorescencia en el lugar del EPR enrollado e hiperfluorescencia marcada en el lugar de la coroides expuesta fig. 1c) [24]. En 2010, Sarraf et al. [24] describieron un ligero defecto en el margen del DEP con un tenue anillo de hiperfluorescencia en la AGF. Esta observación se encontró en desgarros del EPR de grado 1 (mayor diámetro lineal $<200 \mu \mathrm{m}$ ) y representa un defecto microscópico del EPR detectable en las correspondientes imágenes de tomografía de coherencia óptica de dominio espectral (OCT SD). Las lesiones $>200 \mu \mathrm{m}$ se presentan mediante angiografía como defectos hiperfluorescentes ovalados al borde del DEP con transmisión temprana, tinción tardía y un sutil borde interior de bloqueo hipofluorescente. Los desgarros del EPR de mayor tamaño muestran una gran área semilunar de hiperfluorescencia de transmisión temprana con una mancha adyacente de bloqueo hipofluorescente.

Tomografía de coherencia óptica de dominio espectral (OCT SD)

En un típico desgarro del EPR, se distinguen tres áreas distintas en las imágenes de OCT SD. En la primera parte, las bandas externas de la retina muestran una arqui-

Desgarros del EPR tectura normal. En la segunda parte, la retina forma un desprendimiento cupuliforme a partir de la coroides. En esta zona elevada, la OCT SD muestra el borde libre de un EPR distorsionado y contraído que se retrae hacia la NVC. El EPR enrollado provoca hiperreflectividad y un ensombrecimiento posterior intenso, que enmascara por completo la coroides. En la tercera parte, el EPR se encuentra totalmente ausente y la retina neurosensorial aparece aclarada. La OCT SD demuestra un aumento de la señal profunda debido a la monocapa del EPR ausente (fig. 1d). En algunos casos, durante el seguimiento tras el evento agudo, se puede visualizar una reacción exudativa entre la retina neurosensorial y la coroides. En otros casos, la retina neurosensorial permanece directamente sobre la coroides [29].

\section{Mecanismo de formación de los desgarros del EPR}

El desarrollo de los desgarros del EPR puede suceder como un proceso espontáneo o tras láser térmico, terapia fotodinámica o tratamiento anti-VEGF $[2,5,8-11]$. Durante muchos años, varios autores han postulado un mecanismo de desgarro del EPR a partir de la contracción de membranas fibrovasculares [30-33]. Más tarde, en la época de los anti-VEGF, el aumento en la incidencia de los desgarros del EPR en pacientes con DMAE se interpretó como una confirmación de la teoría establecida de fuerzas de tracción que provocan el desgarro. La contracción de las membranas de la NVC ocasiona un encogimiento del EPR, que puede provocar aumento de tensión en la superficie de la cavidad. Durante este proceso, dos fuerzas opuestas actúan en el EPR periférico: las de tracción a partir de la contracción de la NVC y las adhesivas a partir del EPR que todavía está unido. El aumento de la contracción finalmente conlleva a la falla anatómica del EPR en la zona de unión del EPR adherido y desprendido (fig. 2) [29, 34].

Las técnicas de diagnóstico por imagen multimodales proporcionan una percepción mecanicista del desarrollo de los desgarros del EPR a partir de la configuración arquitectónica previa y posterior al desgarro del DEPv. Spaide [35] asumió que el material acumulado bajo el EPR representa una NVC dentro del desprendimiento del epitelio pigmentario de la retina. La contractura de esta NVC adherida a la superficie inferior del EPR aplica la máxima tracción en la zona de unión del EPR adherido y desprendido, que representa el locus minores resistentiae (lugar de menor resistencia) y se dispone perpendicular a la fuerza de contracción de la NVC. La monocapa

Ophthalmologica 2017;238(suppl 1):28-38 DOI: $10.1159 / 000477514$ 

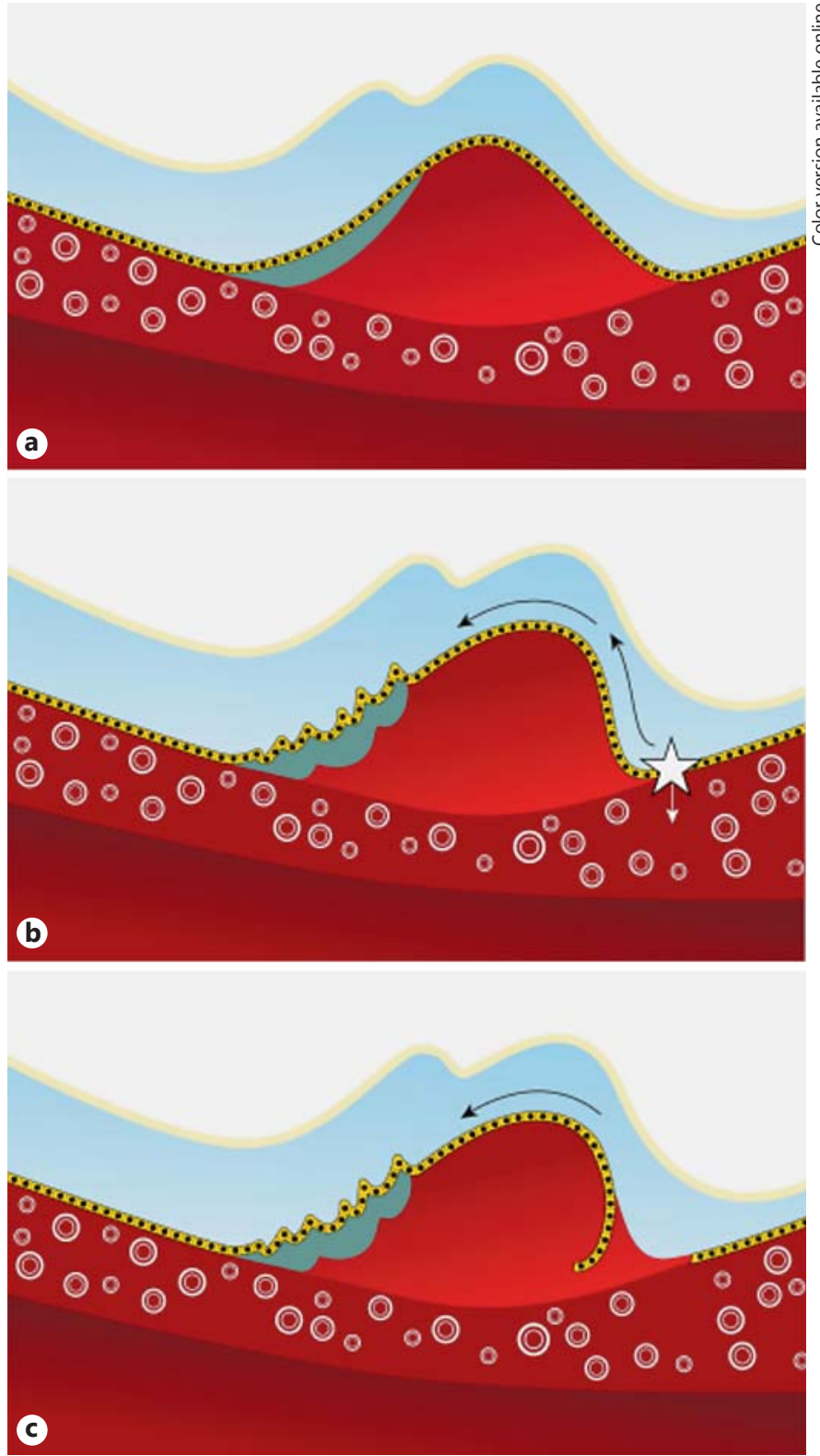

Fig. 2. Ilustración esquemática que muestra el mecanismo propuesto de desgarro del EPR en ojos con degeneración macular neovascular asociada a la edad tras tratamiento anti-VEGF intravitreo. a El DEPv contiene la membrana de la NVC oculta (azul-gris) adherente a la superficie inferior de la monocapa del EPR (capa celular amarilla-negra). b La contracción de la NVC provoca fuerzas de tracción que actúan en el plano del EPR aplicando la mayor tensión mecánica en la zona de unión del EPR adherido y desprendido (estrella). En esta localización actúan dos fuerzas opuestas: fuerzas de tracción a partir de la contracción de la NVC y fuerzas adhesivas a partir del EPR que todavía está unida (flechas). c El aumento de la contracción finalmente conlleva el fracaso anatómico del EPR en la zona de unión del EPR adherido y desprendido. El borde libre del desgarro del EPR se retrae hacia la NVC. Para ver los colores, observe la versión en línea. contraída del EPR permanece en la parte de la NVC, el origen de la contracción, que se refleja en la OCT SD o como hiperautofluorescencia en AFF. Estudios anteriores presentaron una tendencia de los desgarros del EPR a desarrollarse en el borde temporal de la lesión del DEPv. $[36,37]$. Se desconoce la razón por la que el desarrollo de los desgarros del EPR suele ocurrir aquí.

La formación de desgarros del EPR en DEP fibrovasculares, en los que la cavidad de la lesión está completamente rellenada por la membrana de la NVC, es menos frecuente. Esto se debe supuestamente a que las fuerzas de contracción en respuesta a un tratamiento anti-VEGF pueden distribuirse de forma uniforme por toda la lesión del DEP exponiendo la monocapa del EPR a una menor tensión mecánica. En cambio, en un DEP seroso vascularizado, la membrana de la NVC solo rellena parcialmente la cavidad del desprendimiento [34]. Es interesante señalar que los desgarros del EPR en pacientes en tratamiento anti-VEGF tienden a suceder en los primeros 3 meses del comienzo del tratamiento intravítreo, mientras que los desgarros del EPR de comienzo tardío ocurren en pacientes no tratados previamente (naïve) que se someten a terapia fotodinámica [17].

En contraposición a los desgarros del EPR convencionales, Ie et al. [38] hipotetizaron que el mecanismo que conlleva un microdesgarro del EPR se debe a fuerzas hidroestáticas que solas producen una ruptura del borde del EPR. El microdesgarro no debería considerarse un estadio previo del desgarro del EPR ni el comienzo de un desgarro del EPR. Es mucho más probable que los microdesgarros y los desgarros del EPR representen dos entidades independientes basadas en distintos mecanismos etiológicos, tal y como postulan Ie et al. [38] en contraposición a Sarraf et al. [39].

\section{Factores pronósticos}

Dado que un desgarro del EPR normalmente provoca una pérdida grave de la agudeza visual, la identificación de factores de riesgo fiables resulta de gran relevancia desde el punto de vista clínico. Hasta ahora, se han descrito varios marcadores pronósticos de un desgarro inminente del EPR, tales como la altura y el diámetro de la lesión del DEP, líneas hiperreflectivas en imágenes del infrarrojo cercano, una relación pequeña entre el tamaño de la NVC y del DEP, fisuras subretinianas, microdesgarros y duración del DEP.

La altura del DEP se ha reportado como un factor pronóstico de los desgarros por Chan et al. [13]. Ellos repor- 


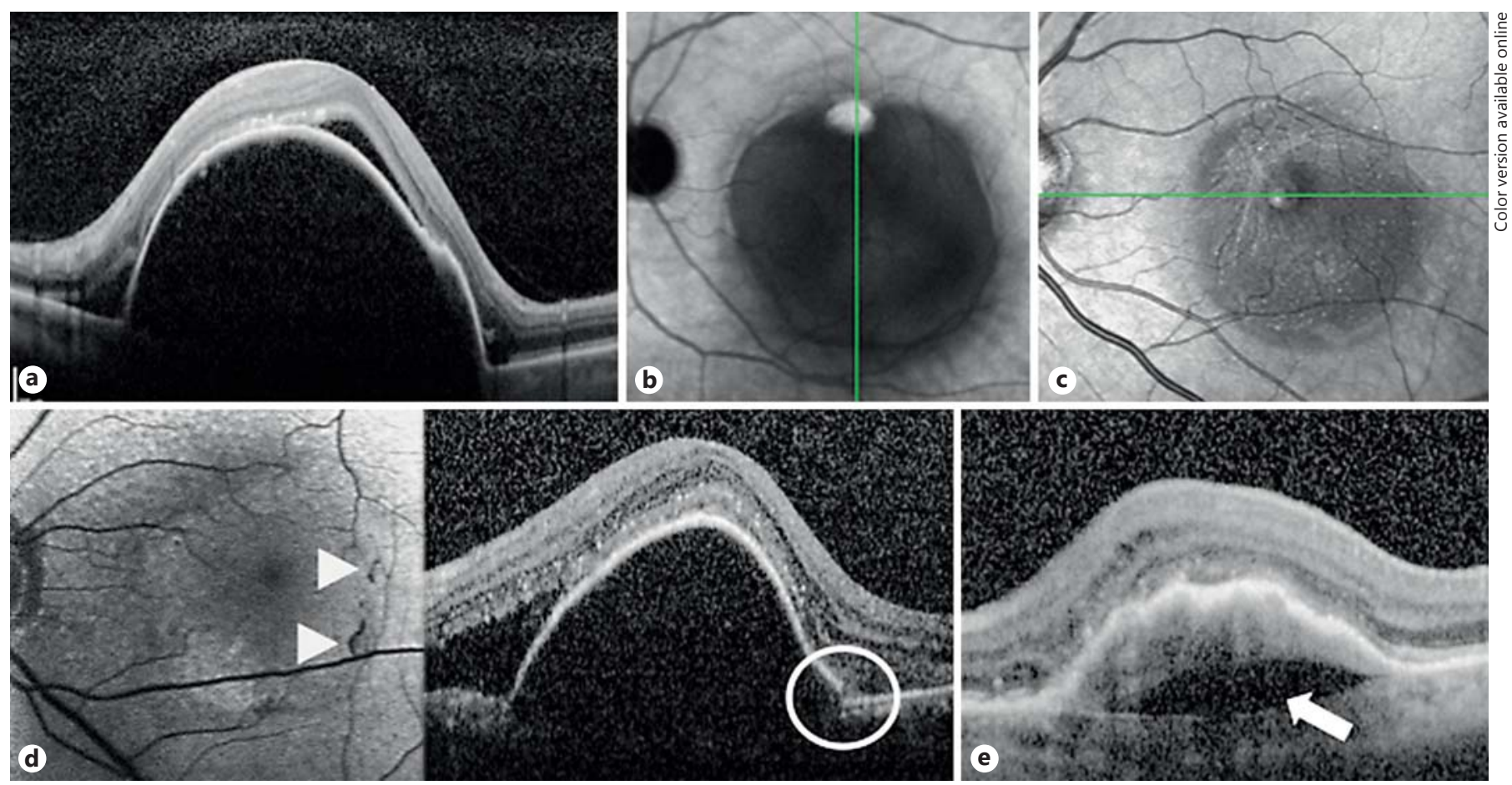

Fig. 3. Factores de riesgo del desgarro del EPR en pacientes con DEPv debido a una DMAE. a Escáner OCT SD de una lesión de DEPv tanto con un gran diámetro como con una gran altura. b Imagen de angiografía con verde de indocianina que ilustra el concepto de una pequeña relación NVC/DEP. La hiperfluorescencia en la parte superior representa una pequeña NVC mientras que la hiperfluorescencia circular representa las grandes dimensiones del DEPv, lo que conlleva una relación muy pequeña. c La imagen del infrarrojo cercano de la oftalmoscopia confocal láser de barrido de otra lesión de DEPv muestra líneas hiperreflectivas que se orig- inan a partir del borde del DEPv que se extienden en forma de embudo por la lesión. d AFF con oftalmoscopia confocal láser de barrido combinada con OCT SD que representa pequeñas hipoautofluorescencias en el borde de un DEPv indicando áreas de microdesgarros (puntas de flecha) que corresponden a defectos microscópicos del EPR (círculo) detectables en la OCT SD a la derecha. e Imágenes de escáner OCT SD de profundidad aumentada que muestra una fisura subretiniana. La flecha apunta a un espacio hiporreflectivo por debajo del tejido neovascular subEPR. taron aumento en la prevalencia de los desgarros del EPR en lesiones de DEP superiores a $400 \mu \mathrm{m}$. Varios otros autores han abordado la cuestión de en qué umbral la altura del DEP se convierte en un factor de riesgo. Doguizi y Ozdek [19] determinaron de forma estadística que 580 $\mu \mathrm{m}$ se podía considerar un umbral de altura del DEP para que exista riesgo de que se produzca un desgarro del EPR. De manera similar, Sarraf et al. [39] describieron una altura de $500 \mu \mathrm{m}$ como factor de riesgo para el posterior desarrollo de un desgarro del EPR; de forma adicional, Leitritz et al. [40] describieron que resultaría más probable de que se produjeran desgarros del EPR por encima de los $400 \mu \mathrm{m}$ de altura. Además, Chiang et al. [20] hipotetizaron que un aumento del área de la superficie y un gran diámetro lineal de un DEP subfoveal representan factores pronósticos para el desarrollo de un desgarro del EPR (fig. 3a). Chan et al. [37] presentaron una tendencia

Desgarros del EPR más sólida al desarrollo de desgarros en casos de DEP que muestran una proporción menor del tamaño de la NVC con respecto al tamaño del DEP (fig. 3b).

La detección de un aumento de las señales de reflectancia en imágenes del infrarrojo cercano mediante oftalmoscopia confocal láser de barrido representa otro marcador pronóstico de desgarro inminente del EPR (fig. 3c). Estas líneas hiperreflectivas, que se originan de forma característica a partir del borde de los DEP y que normalmente se extienden en forma de embudo, corresponden a la localización de la NVC que se observa en la angiografía con verde de indocianina. De manera adicional, las líneas hiperreflectivas en imágenes del infrarrojo cercano se relacionan con pliegues en el EPR detectables en la OCT SD [34]. Estos resultados indican la tensión mecánica provocada tanto por la contracción como por la relajación de la NVC. 
Los microdesgarros del EPR se describieron inicialmente como una fuga en el borde del desprendimiento del EPR por el que pasa la fluoresceína al espacio subretiniano [38]. Más tarde, Sarraf et al. [24] introdujeron un sistema de clasificación de desgarros del EPR definidos en grado 1 como un leve defecto en el margen del DEP con un tenue anillo de hiperfluorescencia en la angiografía, así como un defecto microscópico del EPR detectable en la OCT. Hace poco tiempo, Clemens et al. [41] plantearon considerar los microdesgarros como un factor de riesgo de los desgarros del EPR al presentar un paciente con microdesgarros que desarrolló un desgarro foveal del EPR tras una inyección de anti-VEGF. Supuestamente, los microdesgarros reducen el umbral de resistencia del EPR, y un aumento de la contracción tras tratamiento anti-VEGF puede provocar en última instancia falla anatómica del EPR (fig. 3d).

Recientemente, Mukai et al. [42] describieron la formación de una fisura subretiniana previa al desarrollo de un desgarro del EPR en 3 pacientes, lo que indicaba que esta característica en la OCT SD suponía un posible factor de riesgo (fig. 3e). Esta fisura subretiniana puede ser el resultado de una mayor tensión mecánica en el espacio inferior al EPR

Finalmente, Doguizi y Ozdek [19] presentaron una relación inversa entre la duración del DEP y la formación de un desgarro del EPR. En el grupo de pacientes con desgarro del EPR de su estudio, el DEP se presentó en un periodo de tiempo más corto. Planteaban que una duración corta del DEP significa que el proceso neovascular se encuentra fresco, sin vasos inmaduros. Dado que los vasos inmaduros son más susceptibles a los anti-VEGF, la respuesta a este tratamiento puede resultar más considerable.

\section{Tratamiento en pacientes de alto riesgo}

Se recomienda a los médicos que busquen factores de riesgo del desgarro del EPR demostrados antes de administrar un tratamiento anti-VEGF en pacientes con DEPv por DMAE. Los pacientes con PEDv de alto riesgo se consideran aquellos que poseen uno o más de los factores de riesgo del desgarro del EPR descritos al comienzo o durante el transcurso de un tratamiento anti-VEGF. En el caso de estos pacientes, se recomienda realizar un exhaustivo reconocimiento que incluya OCT SD y AFF después de administrar cada inyección. Si se detectan varios factores de riesgo o si un único factor de riesgo aumenta de forma significativa durante el tratamiento
anti-VEGF, recomendamos interrumpir el tratamiento de inyección, volver a evaluar la lesión del DEP 1 ó 2 semanas más tarde, y volver a administrar la inyección si los signos de contracción de la NVC han disminuido, por ejemplo, si los pliegues del EPR disminuyen o las líneas hiperreflectivas desaparecen. Un régimen adaptado como este puede hacer que el tratamiento anti-VEGF sea más seguro con respecto al desarrollo de desgarros del EPR en pacientes con DEPv de alto riesgo. Por otro lado, el hecho de posponer el tratamiento conlleva el riesgo de que la NVC progrese.

Recientemente, Chan et al. [43] presentaron resultados de un estudio prospectivo en el que se comparaban los resultados de las inyecciones de ranibizumab intravítreo de 0,5 y 2,0 mg para el tratamiento de DEPv. La dosis más alta reportó reducciones más rápidas y una resolución más completa del DEP; no obstante, la incidencia de desgarro del EPR fue superior en este grupo. Los datos indican que el cuanto de las fuerzas de contracción en la lesión del DEP provocada por el agente anti-VEGF debe pensarse con mucho cuidado. Una sobredosis podría fácilmente provocar el desarrollo de un desgarro, en especial en pacientes de alto riesgo.

La mayoría de los datos disponibles sobre desarrollo de un desgarro del EPR después de recibir tratamiento son con bevacizumab y ranibizumab, mientras que hasta ahora solo se disponen de casos clínicos individuales sobre aflibercept [11]. Las secuencias del receptor de aflibercept proporcionan una potente unión al VEGF (140 veces más que ranibizumab), y la molécula posee una actividad de unión intravítrea de 1 mes que excede tanto la de ranibizumab como la de bevacizumab. A diferencia de ambos agentes anti-VEGF, aflibercept se une no solo a todas las isomerasas de la familia A-VEGF y B-VEGF, sino también al factor de crecimiento placentario [44]. Se desconoce la relevancia clínica de estas diferencias farmacológicas. No obstante, se podría proponer utilizar determinados medicamentos para determinados tipos de DEP. Teniendo en cuenta la mayor potencia de la contracción de la NVC del aflibercept, resultaría razonable preferir el uso de ranibizumab en pacientes con DEPv de alto riesgo y aflibercept en casos de pacientes con DEP de bajo riesgo fibrovascular. Hubo un caso clínico que describió el desarrollo de un desgarro del EPR tras dos inyecciones de aflibercept en un paciente con PEDv que había sido tratado previamente con ranibizumab [45]. Sin embargo, hoy en día no se dispone de datos sobre esta situación. Actualmente, un grupo de estudio está reclutando pacientes para investigar de forma prospectiva el efecto de aflibercept en pacientes con PEDv sobre la in-
34

Ophthalmologica 2017;238(suppl 1):28-38 DOI: $10.1159 / 000477514$
Clemens/Eter 
cidencia del desarrollo de desgarros del EPR. Además de cuál sería el agente anti-VEGF más adecuado en el caso de pacientes de alto riesgo, otra duda sin resolver es si la adherencia a un determinado régimen terapéutico podría resultar beneficioso en relación con el desarrollo de desgarros en este grupo de pacientes. En cualquier caso, y en particular en el de ojos con DEP de alto riesgo, los pacientes deben ser informados sobre el riesgo de que se desarrollen desgarros durante el tratamiento anti-VEGF.

\section{Tratamiento tras la formación de desgarros}

Existe cierta controversia entre los médicos sobre los criterios terapéuticos tras la formación de desgarros del EPR. Un análisis retrospectivo de datos de tres ensayos clínicos en fase III aleatorizados y multicéntricos de ranibizumab para el tratamiento de DMAE neovascular mostró que, entre los pacientes que padecían un desgarro del EPR, los tratados con ranibizumab tendían a conseguir mayor mejoría de la agudeza visual [46]. Doguizi y Ozdek [19] demostraron unos resultados estables de agudeza visual en 28 pacientes con tratamiento anti-VEGF continuado tras la formación de un desgarro del EPR en un periodo medio de seguimiento de 20,6 meses. La media de inyecciones anti-VEGF fue 4,1 durante este periodo de seguimiento. La agudeza visual final fue peor en el caso de desgarros mayores del EPR (grados 3 y 4 ) al compararlos con desgarros más pequeños (grados 1 y 2), lo que coincide con los resultados de Sarraf et al. [24]. Coco et al. presentaron resultados funcionales similares [47]. También se han presentado mejoras de la agudeza visual en pacientes con desgarros del EPR espontáneos que posteriormente se han sometido a tratamiento anti-VEGF [48]. Recientemente, Bartels et al. [49] presentaron un caso de mejora funcional significativa en microperimetría y morfología tras continuar un tratamiento antiVEGF durante más de 3 años debido a la presencia de fluido subretiniano e intrarretiniano persistente tras la formación de un desgarro.

Hoy en día, no existe ningún ensayo clínico prospectivo al respecto; sin embargo, sí hay un ensayo multicéntrico intervencional en el que se está reclutando a pacientes y se investiga el efecto de las inyecciones intravítreas fijas mensuales de ranibizumab en la agudeza visual y la morfología de la retina en el marco de un estudio de 12 meses en 30 pacientes con desgarro del EPR debido a una degeneración macular neovascular asociada a la edad (ClinicalTrials.gov identificador: NCT01914159). En la actualidad, la mayoría de los expertos en retina reco-

Desgarros del EPR miendan tratamiento anti-VEGF continuado tras el desarrollo de un desgarro del EPR según la presencia de actividad de la enfermedad, como por ejemplo fluido subretiniano y teniendo en cuenta que se espera un beneficio funcional o una estabilización.

No obstante, debe evaluarse con cuidado la reinyección tras un desgarro del EPR, pues el área del desgarro puede aumentar de forma significativa durante el tratamiento anti-VEGF. Asao et al. [50] evaluaron los efectos de un tratamiento anti-VEGF adicional en 10 ojos con desgarro del EPR tras recibir tratamiento durante 12 meses. Observaron un aumento del tamaño del área del desgarro del EPR del $>20 \%$ en la mitad de los ojos. Clemens et al. [26] presentaron 3 pacientes que inicialmente mostraban un desgarro del EPR multilobular, revelando un crecimiento significativo del desgarro con afectación foveal tras recibir otra inyección anti-VEGF. Por otro lado, el mismo estudio mostró que los defectos de los desgarros del EPR unilobulares permanecían estables durante un periodo de seguimiento de 6 meses. Presumiblemente, parece que los filamentos del EPR entre los lóbulos del desgarro del EPR evitan la progresión del desgarro en el caso de desgarros del EPR multilobulares. El hecho de que se midan las áreas de desgarro del EPR en estos pacientes puede tener consecuencias terapéuticas. Si el área de desgarro del EPR en lesiones multilobulares aumenta, el tratamiento intravítreo con anti-VEGF debería posponerse, y tan solo se debería retomar si aumentan más los signos de actividad de la enfermedad. De forma adicional, en pacientes con pequeñas áreas de desgarro del EPR fuera de la fóvea y con lesiones inactivas de la NVC, resulta más razonable realizar seguimientos cercanos que volver a administrar una inyección inmediata.

Mukai et al. [51] observaron dos tipos de procesos de reparación tras el desarrollo de desgarros del EPR en un estudio pequeño de pruebas de diagnóstico por imagen de 10 pacientes. En primer lugar, el fluido subretiniano persistente que se observó tras el desgarro parece conllevar la posterior reparación con un tejido proliferativo engrosado en el área donde se perdió el EPR. En segundo lugar, si se consigue una resolución completa del fluido subretiniano tras el desarrollo del desgarro del EPR, la retina exterior aparece completamente unida a la membrana de Brunch sin que exista una penetración de tejido proliferativo en ella. Estas observaciones sugieren un efecto beneficioso sobre la morfología macular como consecuencia de un tratamiento anti-VEGF continuado tras el desarrollo de un desgarro del EPR. Sin embargo, todavía queda por demostrar si los dos mecanismos de reparación conllevan resultados funcionales diferentes.

Ophthalmologica 2017;238(suppl 1):28-38 DOI: $10.1159 / 000477514$ 
Mendis y Lois [52] presentaron un estudio longitudinal de AFF en 14 pacientes con desgarro del EPR e informaron de una reconstrucción de la superficie del área desbridada del EPR en 10 ojos y una progresión de pérdida celular del EPR en 4 ojos. En el primer grupo, la recuperación de la señal de AFF sucedió de forma centrípeta, desde el borde del área del defecto hacia el centro, a excepción del borde en el que se encontraba el EPR enrollado.

Estudios previos demostraron que la membrana basal del EPR es más propicia a la reconstrucción de la superficie del EPR, pues sirve como estructura de andamiaje, que la capa de colágeno más profunda de la membrana de Brunch, lo que indica que la profundidad del plano de escisión puede afectar al alcance de la reconstrucción de la superficie del EPR. Varios estudios experimentales mostraron que las células del EPR realizan una acción de repoblación desde el borde hacia el centro, en lugar de hacerlo a partir de las células residuales que permanecen en el área desbridada $[53,54]$.

Caramoy et al. [55] analizaron la remodelación del tejido del EPR en los desgarros en función de la AFF y las imágenes de la OCT SD y observaron pruebas de reconstrucción de la superficie en el caso de los pequeños desgarros del EPR, mientras que la migración y proliferación del EPR puede que no suceda en el plano correcto de los desgarros. Se está desarrollando y evaluando el trasplante de EPR con células madre embrionarias humanas y EPR de células madre pluripotentes inducidas como tratamiento sustitutivo celular para la DMAE. Resulta necesario que se lleven a cabo más estudios para evaluar el potencial de restablecer algunas de las funciones perdidas del EPR en pacientes que presentan desgarros del EPR [56].

\section{Conclusiones}

Debido al creciente número de inyecciones antiVEGF intravítreas en pacientes con DMAE, ha aumentado la incidencia en la formación de desgarros del EPR. Por ello, los médicos deben estar informados de las posibles estrategias para prevenir el desarrollo de desgarros del EPR durante un tratamiento anti-VEGF y tratar a los pacientes después de que se formen. Un régimen terapéutico adaptado en casos de DEPv de alto riesgo puede evitar que los pacientes desarrollen desgarros del EPR. Tras la formación de un desgarro del EPR, se recomienda llevar a cabo un tratamiento anti-VEGF mientras los signos de actividad estén presentes. El diagnóstico por imagen multimodal durante un tratamiento anti-VEGF en pacientes de alto riesgo representa el instrumento clave para detectar factores de riesgo y hacer más seguro el tratamiento anti-angiogénico. Los estudios que se realicen en el futuro deben tratar varias cuestiones, tales como qué agente anti-VEGF resulta más adecuado para el tratamiento de pacientes con DEP de alto riesgo, qué régimen terapéutico es más beneficioso tras el tratamiento de desgarros del EPR, y cuánto pueden mejorar las técnicas de diagnóstico por imagen de la retina para visualizar factores de riesgo de desgarros del EPR lo antes posible e identificar nuevas características que indiquen un desgarro inminente.

\section{Declaración de situación}

C.R. Clemens está asociado con Heidelberg Engineering, Novartis y Bayer; N. Eter está asociado con Heidelberg Engineering, Novartis, Bayer, Sanofi Aventis, Allergan y Bausch y Lomb.

\section{Referencias bibliográficas}

1 Casswell AG, Kohen D, Bird AC: Retinal pigment epithelial detachments in the elderly: classification and outcome. Br J Ophthalmol 1985;69:397-403.

2 Yeo JH, Marcus S, Murphy RP: Retinal pigment epithelial tears. Patterns and prognosis. Ophthalmology 1988;95:8-13.

3 Pauleikhoff D, Löffert D, Spital G, Rader-macher M, Dohrmann J, Lommatzsch A, Bird AC: Pigment epithelial detachment in the elderly. Clinical differentiation, natural course and pathogenetic implications. Graefes Arch Clin Exp Ophthalmol 2002;240:533-538.

4 Hoskin A, Bird AC, Sehmi K: Tears of detached retinal pigment epithelium. Br J Ophthalmol 1981;65:417-422.
5 Gelisken F, Inhoffen W, Partsch M, Schneider U, Kreissig I: Retinal pigment epithelial tear after photodynamic therapy for choroidal neovascularization. Am J Ophthalmol 2001; 131:518-520.

6 Gass JD: Retinal pigment epithelial rip during krypton red laser photocoagulation. Am J Ophthalmol 1984;98:700-706.

7 Thompson JT: Retinal pigment epithelial tear after transpupillary thermotherapy for choroidal neovascularization. Am J Ophthalmol 2001;131:662-664.

8 Singh RP, Sears JE: Retinal pigment epithelial tears after pegaptanib injection for exudative age-related macular degeneration. Am J Ophthalmol 2006;142:160-162.
9 Shah CP, Hsu J, Garg SJ, Fischer DH, Kaiser $\mathrm{R}$ : Retinal pigment epithelial tear after intravitreal bevacizumab injection. Am J Ophthalmol 2006;142:1070-1072.

10 Carvounis PE, Kopel AC, Benz MS: Retinal pigment epithelium tears following ranibizumab for exudative age-related macular degeneration. Am J Ophthalmol 2007;143:504505.

11 Saito M, Kano M, Itagaki K, Oguchi Y, Sekiryu $\mathrm{T}$ : Retinal pigment epithelium tear after intravitreal aflibercept injection. Clin Ophthalmol 2013;7:1287-1289. 
12 Meyer CH, Mennel S, Schmidt JC, Kroll P: Acute retinal pigment epithelial tear following intravitreal bevacizumab (Avastin) injection for occult choroidal neovascularisation secondary to age related macular degeneration. Br J Ophthalmol 2006;90:1207-1208.

13 Chan CK, Abraham P, Meyer CH, Kokame GT, Kaiser PK, Rauser ME, Gross JG, Nuthi AS, Lin SG, Daher NS: Optical coherence tomography-measured pigment epithelial detachment height as a predictor for retinal pigment epithelial tears associated with intravitreal bevacizumab injections. Retina 2010;30: 203-211.

14 Chang LK, Flaxel CJ, Lauer AK, Sarraf D: RPE tears after pegaptanib treatment in age-related macular degeneration. Retina 2007;27: 857-863.

15 Clemens CR, Wolf A, Alten F, Milojcic C, Eter $\mathrm{N}$ : Monthly treatment of ranibizumab in vascular pigment epithelium detachment due to age-related macular degeneration. ARVO 2015; Poster 2838 - C0066.

16 Sarraf D, Chan C, Rahimy E, Abraham P: Prospective evaluation of the incidence and risk factors for the development of RPE tears after high- and low-dose ranibizumab therapy. Retina 2013;33:1551-1557.

17 Guber J, Praveen A, Saeed MU: Higher incidence of retinal pigment epithelium tears after ranibizumab in neovascular age-related macular degeneration with increasing pigment epithelium detachment height. Br J Ophthalmol 2013;97:1486-1487.

18 Smith BT, Kraus CL, Apte RS: Retinal pigment epithelial tears in ranibizumab-treated eyes. Retina 2009;29:335-339.

19 Doguizi S, Ozdek S: Pigment epithelial tears associated with anti-VEGF therapy: incidence, long-term visual outcome, and relationship with pigment epithelial detachment in age-related macular degeneration. Retina 2014;34:1156-1162.

20 Chiang A, Chang LK, Yu F, Sarraf D: Predictors of anti-VEGF-associated retinal pigment epithelial tear using FA and OCT analysis. Retina 2008;28:1265-1269.

21 Wong LJ, Desai RU, Jain A, et al: Surveillance for potential adverse events associated with the use of intravitreal bevacizumab for retinal and choroidal vascular disease. Retina 2008; 28:1151-1158.

22 Wolf A, Rüping J, Neubauer AS, Mayer W, Ulbig M, Haritoglou C, Holz FG, Eter N, Kampik A: Alterations of vascular pigment epithelium detachments associated with agerelated macular degeneration during upload with intravitreal ranibizumab. Retina 2013; 33:1843-1849.

23 Mennel S, Callizo J, Schmidt JC, Meyer CH: Acute retinal pigment epithelial tear in the untreated fellow eye following repeated bevacizumab (Avastin) injections. Acta Ophthalmol Scand 2007;85:689-691.

24 Sarraf D, Reddy S, Chiang A, Yu F, Jain A: A new grading system for retinal pigment epithelial tears. Retina 2010;30:1039-1045.
25 von Rückmann A, Fitzke FW, Bird AC: Distribution of fundus autofluorescence with a scanning laser ophthalmoscope. Br J Ophthalmol 1995;79:407-412.

26 Clemens CR, Alten F, Baumgart C, Heiduschka P, Eter N: Quantification of retinal pigment epithelium tear area in age-related macular degeneration. Retina 2014;34:24-31.

27 Caramoy A, Fauser S, Kirchhof B: Fundus autofluorescence and spectral domain optical coherence tomography findings suggesting tissue remodelling in retinal pigment epithelium tear. Br J Ophthalmol 2010;96:12111216.

28 Bindewald A, Schmitz-Valckenberg S, Jorzik JJ, Dolar-Szczasny J, Sieber H, Keilhauer C, Weinberger AW, Dithmar S, Pauleikhoff D, Mansmann U, WolfS, Holz FG: Classification of abnormal fundus autofluorescence patterns in the junctional zone of geographic atrophy in patients with age related macular degeneration. Br J Ophthalmol 2005;89:874878.

29 Nagiel A, Freund KB, Spaide RF, Munch IC, Larsen M, Sarraf D: Mechanism of retinal pigment epithelium tear formation following intravitreal anti-vascular endothelial growth factor therapy revealed by spectral-domain optical coherence tomography. Am J Ophthalmol 2013;156:981-988.

30 Chuang EL, Bird AC: The pathogenesis of tears of the retinal pigment epithelium. Am J Ophthalmol 1988;105:285-290.

31 Gass JDM: Pathogenesis of tears of the retinal pigment epithelium. Br J Ophthalmol 1984; 68:513-519.

32 Toth CA, Pasquale AC III, Graichen DF: Clinicopathologic correlation of spontaneous retinal pigment epithelial tears with choroidal neovascular membranes in age-related macular degeneration. Ophthalmology 1995;102: 272-277.

33 Lafaut BA, Aisenbrey S, Vanden Broecke C, Krott R, Jonescu-Cuypers CP, Reynders S, Bartz-Schmidt KU: Clinicopathological correlation of retinal pigment epithelial tears in exudative age-related macular degeneration: pretear, tear, and scarred tear. Br J Ophthalmol 2001;85:454-460.

34 Clemens CR, Bastian N, Alten F, Milojcic C, Heiduschka P, Eter N: Prediction of retinal pigment epithelial tear in serous vascularized pigment epithelium detachment. Acta Ophthalmol 2014;92:50-56.

35 Spaide RF: Enhanced depth imaging optical coherence tomography of retinal pigment epithelial detachment in age-related macular degeneration. Am J Ophthalmol 2009;147: 644-652.

36 Decker WL, Sanborn GE, Ridley M, Annesley WH Jr, Sorr EM: Retinal pigment epithelial tears. Ophthalmology 1983;90:507-512.
37 Chan CK, Meyer CH, Gross JG, Abraham P, Nuthi AS, Kokame GT, Lin SG, Rauser ME, Kaiser PK: Retinal pigment epithelial tears after intravitreal bevacizumab injection for neovascular age-related macular degeneration. Retina 2007;27:541-551.

38 Ie D, Yannuzzi LA, Spaide RF, Woodward KP, Singerman LJ, Blumenkranz MS: Microrips of the retinal pigment epithelium. Arch Ophthalmol 1992;110:1443-1449.

39 Sarraf D, Chan C, Rahimy E, Abraham P: Prospective evaluation of the incidence and risk factors for the development of RPE tears after high- and low-dose ranibizumab therapy. Retina 2013;33:1551-1557.

40 Leitritz M, Gelisken F, Inhoffen W, Voelker $\mathrm{M}$, Ziemssen F: Can the risk of retinal pigment epithelium tears after bevacizumab treatment be predicted? An optical coherence tomography study. Eye 2008;22:1504-1507.

41 Clemens CR, Alten F, Eter N: Reading the signs: microrips as a prognostic sign for impending RPE tear development. Acta Ophthalmol 2015;93:e600-e602.

42 Mukai R, Sato T, Kishi S: Precursor stage of retinal pigment epithelial tear in age-related macular degeneration. Acta Ophthalmol 2014;92:407-408.

43 Chan CK, Abraham P, Sarraf D, Nuthi AS, Lin SG, McCannel CA: Earlier therapeutic effects associated with high dose $(2.0 \mathrm{mg})$ ranibizumab for treatment of vascularized pigment epithelial detachments in age-related macular degeneration. Eye 2015;29:80-87.

44 Stewart MW, Rosenfeld PJ: Predicted biological activity of intravitreal VEGF trap. Br J Ophthalmol 2008;92:667-668.

45 Bertelmann T, Sekundo W, Wenner Y: Tear in the retinal pigment epithelium by intravitreal injection of aflibercept. Ophthalmologe 2014;111:775-777.

46 Cunningham ET Jr, Feiner L, Chung C, Tuomi L, Ehrlich JS: Incidence of retinal pigment epithelial tears after intravitreal ranibizumab injection for neovascular age-related macular degeneration. Ophthalmology 2011; 118:2447-2452.

47 Coco RM, Sanabria MR, Hernandez AG, Fernández Muñoz M: Retinal pigment epithelium tears in age-related macular degeneration treated with antiangiogenic drugs: a controlled study with long follow-up. Ophthalmologica 2012;228:78-83.

48 Lesniak SP, Fine HF, Prenner JL, Roth DB: Long-term follow-up of spontaneous retinal pigment epithelium tears in age-related macular degeneration treated with anti-VEGF therapy. Eur J Ophthalmol 2011;21:73-76.

49 Bartels S, Barrelmann A, Book B, Heimes B, Gutfleisch M, Spital G, Pauleikhoff D, Lommatzsch A: Tear in retinal pigment epithelium under anti-VEGF therapy for exudative age-related macular degeneration: function recovery under intensive therapy. Ophthalmologe 2014;111:460-464. 
50 Asao K, Gomi F, Sawa M, Nishida K: Additional anti-vascular endothelial growth factor therapy for eyes with a retinal pigment epithelial tear after the initial therapy. Retina 2014; 34:512-518.

51 Mukai R, Sato T, Kishi S: Repair mechanism of retinal pigment epithelial tears in age-related macular degeneration. Retina 2015;35: 473-480.

52 Mendis R, Lois N: Fundus autofluorescence in patients with retinal pigment epithelial (RPE) tears: an in-vivo evaluation of RPE resurfacing. Graefes Arch Clin Exp Ophthalmol 2014;252:1059-1063.
53 Leonard DS, Zhang XG, Panozzo G, Sugino IK, Zarbin MA: Clinicopathological correlation of localized retinal pigment epithelium debridement. Invest Ophthalmol Vis Sci 1997;38:1094-1109.

54 Del Priore LV, Kuo YH, Tezel TH: Age related changes in human RPE cell density and apoptosis proportion in situ. Invest Ophthalmol Vis Sci 2002;43:3312-3318.
55 Caramoy A, Kirchhof B, Fauser S: Morphological versus functional photoreceptor viability of retinal pigment epithelium tears. Acta Ophthalmol 2012;90:328-329.

56 Stanzel BV, Liu Z, Somboonthanakij S, Wongsawad W, Brinken R, Eter N, Corneo B, Holz FG, Temple S, Stern JH, Blenkinsop TA: Human RPE stem cells grown into polarized RPE monolayers on a polyester matrix are maintained after grafting into rabbit subretinal space. Stem Cell Reports 2014;2:64-77. 